\title{
Cross-Field Transport by Instabilities and Blobs in a Magnetized Toroidal Plasma
}

\author{
M. Podestà, ${ }^{*}$ A. Fasoli, B. Labit, I. Furno, P. Ricci, F. M. Poli, ${ }^{\dagger}$ A. Diallo, S. H. Müller, ${ }^{\ddagger}$ and C. Theiler \\ Centre de Recherches en Physique des Plasmas-Ecole Polytechnique Fédérale de Lausanne (EPFL), \\ Association EURATOM-Confédération Suisse, CH-1015 Lausanne, Switzerland
}

(Received 2 March 2008; published 22 July 2008)

\begin{abstract}
The mechanisms for anomalous transport across the magnetic field are investigated in a toroidal magnetized plasma. The role of plasma instabilities and macroscopic density structures (blobs) is discussed. Examples from a scenario with open magnetic field lines are shown. A transition from a main plasma region into a loss region is reproduced. In the main plasma, which includes particle and heat source locations, the transport is dominated by the fluctuation-induced particle and heat flux associated with a plasma instability. On the low-field side, the cross-field transport is ascribed to the intermittent ejection of macroscopic blobs propagating toward the outer wall. It is shown that instabilities and blobs represent fundamentally different mechanisms for cross-field transport.
\end{abstract}

DOI: 10.1103/PhysRevLett.101.045001

It is widely recognized that the transport of particles and heat across the magnetic field in magnetically confined plasmas is anomalous, i.e., much larger than the transport induced by collisional processes [1]. Similar statistical properties, observed in different physical systems and/or for a variety of experimental conditions [2], suggest that the mechanisms responsible for the anomalous cross-field transport may have a common fundamental character. In particular, the possible relationship with low-frequency electrostatic instabilities has been suggested [3]. Correlated fluctuations of density and potential associated with unstable modes can directly drive a cross-field flux [4]. In addition, losses are partly due to the ejection of blobs, i.e., macroscopic density perturbations stretched along the magnetic field [5]. Theoretical [6-8] and experimental $[9,10]$ investigations are shedding light upon the mechanisms governing the blob dynamics, hence the associated transport $[1,11]$. However, despite the fundamental difference in the properties of instabilities and blobs, no clear distinction is usually done between their contributions to the total transport, generically interpreted in terms of a turbulent flux.

In this Letter, we address the question of the different contributions to the cross-field transport in a simple magnetized toroidal plasma from instabilities and blobs. It is shown that the associated mechanisms for cross-field transport present fundamental differences. Therefore, the corresponding transport rates should be measured through complementary techniques to fully characterize the turbulent flux. For the scenario investigated herein, a main plasma and an edge region, connected by a transition region, can be clearly separated [12]. An interchange instability develops in the main plasma [13]. Blobs originate from the instability in the transition region, then propagate toward the outer wall across the magnetic field $[12,14]$. Although $\gtrsim 90 \%$ of the total losses on TORPEX plasmas are due to losses along the open magnetic field lines [15], in this Letter we focus on cross-field transport induced by perturbations of the plasma parameters.
PACS numbers: 52.25.Fi, 52.55.Dy, 52.55.Hc

In general, the instantaneous particle flux is $n \mathbf{v}$, where $n$ is the local plasma density and $\mathbf{v}$ is assumed to be dominated by the $\mathbf{E} \times \mathbf{B}$ velocity [16] ( $\mathbf{E}$ and $\mathbf{B}$ are the electric and magnetic fields). A net transport rate results from the time average of the flux $n \mathbf{v}$. As different phenomena may lead to perturbations with specific spatial and temporal scales, calculating the net transport rate requires, in practice, identifying the correct expressions for $n$ and $\mathbf{v}$. Two cases can be readily separated, depending on whether perturbations can be described through the local (singlepoint) quantities $n(\mathbf{x}, t), \mathbf{v}(\mathbf{x}, t)$. Here $\mathbf{x}=r \hat{\mathbf{e}}_{r}+z \hat{\mathbf{e}}_{z}$ is the spatial coordinate, with $r$ along the major radius and $z$ in the vertical direction. The first case applies to instabilities, characterized by a linear dispersion relation $\omega(\mathbf{k})$, where $\omega / 2 \pi$ and $\mathbf{k}$ indicate frequency and wave number. The second case includes macroscopic and intermittent events such as blobs, for which a local dispersion relation cannot be determined.

In the presence of instabilities which are not in a fully developed turbulent state, the local plasma response can be recovered from $\omega(\mathbf{k})$. The transport rate is obtained by averaging the instantaneous flux over a period much larger than that associated with the characteristic frequency. Retaining only first-order perturbations around timeaveraged values, the fluctuation-induced particle and heat transport rates are [17]

$$
\begin{gathered}
\boldsymbol{\Gamma}=\left\langle n_{1} \mathbf{v}_{E_{1} \times B}\right\rangle_{t}, \\
\mathbf{Q}=\frac{3}{2} n_{0}\left\langle T_{1} \mathbf{v}_{E_{1} \times B}\right\rangle_{t}+\frac{3}{2} T_{0} \boldsymbol{\Gamma} .
\end{gathered}
$$

$T_{e}$ is the electron temperature, and $\langle\ldots\rangle_{t}$ denotes the time average. Subscripts 0 and 1 indicate the time-averaged and fluctuating parts. In the electrostatic limit $\mathbf{v}_{E_{1} \times B}=$ $-\nabla \Phi_{1} \times \mathbf{B}_{0} / B_{0}^{2}$, where $\Phi$ is the plasma potential. For fluctuations satisfying a linear dispersion relation, the spectral properties of $\boldsymbol{\Gamma}$ can be unfolded from a Fourier decomposition of Eq. (1) [16]. For the radial particle transport is 


$$
\Gamma_{r}=-\operatorname{Re}\left[\frac{i}{\pi B} \int_{0}^{\infty} k_{z} \gamma e^{i \alpha_{n, \Phi}} \sqrt{P_{n} P_{\Phi}} d \omega\right] .
$$

For each frequency component, $k_{z}(\omega)$ is the wave number along $z, \gamma(\omega)$ and $\alpha_{n, \Phi}(\omega)$ the coherence and the phase angle between $n_{1}$ and $\Phi_{1}$, and $P_{n}, P_{\Phi}$ their power spectral densities. Re indicates the real part.

In the presence of macroscopic blobs, the local (singlepoint) $n$ and $v$ are not sufficient to fully characterize the associated transport. The behavior over spatial scales of the order of the blob size must be considered. Following Ref. [18], no assumptions on the mechanisms determining the flux are invoked, and any event which leads to a macroscopic density variation is included in the evaluation of the flux. The particle transport associated with an event is calculated by reconstructing the dynamics of plasma structures, i.e., macroscopic bounded regions where the density deviates from its time-averaged value

$$
\boldsymbol{\Gamma}_{\text {str }}=\left\langle\bar{n}_{\text {str }} \mathbf{v}_{\text {str }}\right\rangle
$$

Here $\bar{n}_{\text {str }}$ and $\mathbf{v}_{\text {str }}$ are the average structure density and velocity. Brackets indicate the average over the ensemble of structures moving through each specific location. More details on the method are found in Ref. [18]. Note that, when structures represent intermittent events such as blobs, an additional factor, given by the typical duration of an event times the number of events per unit time, must be included to compute the time-averaged transport from Eq. (4) [12].

In the following, the two approaches are applied to a specific experimental scenario achieved on TORPEX [19], a toroidal device with major and minor radius of 1 and $0.2 \mathrm{~m}$. The helical magnetic field results from the superposition of a dominant toroidal component of $76 \mathrm{mT}$ on the axis, and a small vertical component of $2.3 \mathrm{mT}$. Plasmas are produced from hydrogen at a base pressure of $3.5 \times$ $10^{-5}$ mbar by $400 \mathrm{~W}$ of microwave power, injected from the low-field side at $2.45 \mathrm{GHz}$, in the electron-cyclotron frequency range. The main diagnostics are electrostatic Langmuir probes. The sampling rate of $250 \mathrm{kHz}$ provides a good over-sampling of the data over the typical timescales of perturbations observed on TORPEX [19]. The time-averaged profiles of $n, \Phi$ and $\mathbf{v}_{E \times B}$, measured through swept Langmuir probes, are shown in Figs. 1(a) and 1(b). The plasma profiles are vertically elongated, with a strong pressure gradient for $r>-4 \mathrm{~cm}$. The inhomogeneous profile of $\Phi$ results in a sheared $\mathbf{E} \times \mathbf{B}$ velocity field, with a maximum magnitude of $\sim 1.5 \mathrm{~km} / \mathrm{s}$ at $r \approx 2 \mathrm{~cm}$. In that region, plasma fluctuations are dominated by a narrow-band interchange mode [13] [20] at $\approx 4 \mathrm{kHz}$, which develops in the region of bad curvature of $\mathbf{B}$ and strong plasma pressure gradient. Blobs are observed in the source-free region, $r>5 \mathrm{~cm}$. More details on similar scenarios, including the mechanisms for blob generation and the statistical properties of the fluctuations, are given in Refs. $[12,14,20]$.
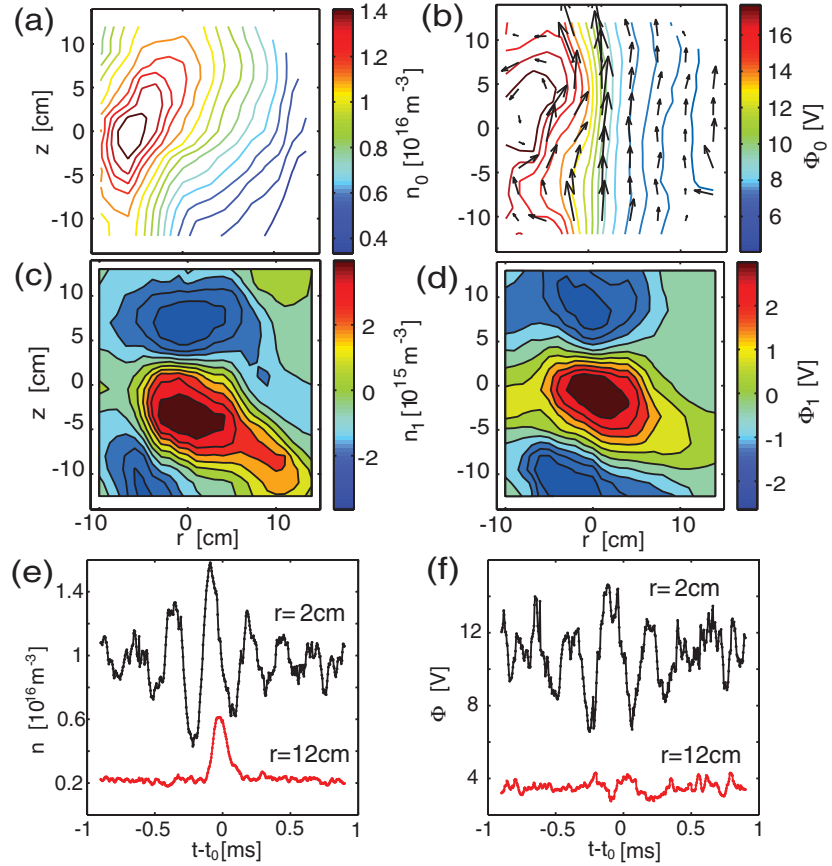

FIG. 1 (color). (a)-(b) Time-averaged profiles of $n, \Phi$ and $\mathbf{v}_{E \times B}$ velocity field (arrows in frame b). The length of the arrows is $\propto\left|\mathbf{v}_{E \times B}\right|$. (c-d) Snapshots of $n_{1}$ and $\Phi_{1}$, measured through the CS technique. (e-f) Reconstructed time traces of $n$ and $\Phi$ at $z=-5 \mathrm{~cm}$, for $r=2 \mathrm{~cm}$ and $r=12 \mathrm{~cm}$. $t_{0}$ is the time at which a blob is detected on the reference probe.

The radial particle transport at midplane is measured from Fourier analysis [Eq. (3)] by a three-tip probe. The effects of finite $T_{e}$ fluctuations, independently measured with a triple probe, are included. The two-dimensional $\boldsymbol{\Gamma}$ and $\mathbf{Q}$ are calculated from the time series of $n, T_{e}$ and $\Phi$ reconstructed from conditionally sampled (CS) data from swept Langmuir probes $[15,20,21]$. The spatial resolution of the reconstructed CS signals is $1 \mathrm{~cm}$ radially and $1.8 \mathrm{~cm}$ vertically. The reference probe for the CS is at $r=14 \mathrm{~cm}$ on the midplane, in order to reconstruct at the same time the dynamics of the instability and of the average blob [20]. The threshold defining a trigger event is 4 times the standard deviation of the reference signal. Examples of the reconstructed 2D profiles of $n_{1}$ and $\Phi_{1}$, along with typical time traces, are presented in Figs. 1(c)-1(f). The fluctuation-induced particle transport, computed from CS data, is mostly directed outward from the region of maximum density (Fig. 2). The heat flux is $\mathbf{Q} \propto \boldsymbol{\Gamma}$ (see inset in Fig. 2) due to the fact that $T_{e, 1}$ and $\Phi_{1}$ are in phase. Heat is passively convected by the net particle flux, rather than driven by electron temperature fluctuations themselves, resulting in a negligible contribution from conduction [17]. The results obtained from the CS and Fourier analysis are compared in Fig. 3(a) for the radial component at midplane. In the main plasma there is a qualitative agreement between the two techniques. The quantitative discrepancy by a factor $\sim 5$ is due to the averages implied by the CS method [22]. The Fourier analysis reveals that the 


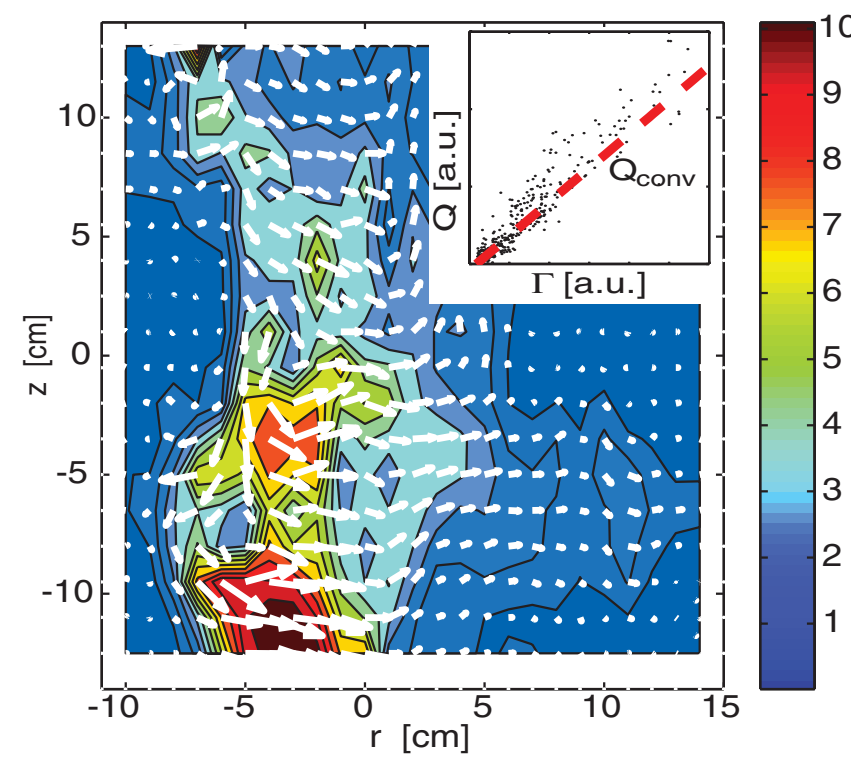

FIG. 2 (color). 2D profile of the fluctuation-induced particle flux (units: $10^{17} \mathrm{~m}^{-2} \mathrm{~s}^{-1}$ ), reconstructed from CS data, associated with an interchange mode at $\approx 4 \mathrm{kHz}$. The inset highlights the character of $\mathbf{Q} \propto \boldsymbol{\Gamma}$, with the dashed line representing a least-square fit of the contribution $3 / 2 T_{e, 0} \Gamma$ [cf. Eq. (2)].

coherence between $n_{1}$ and $\Phi_{1}$ vanishes for all frequencies in the source-free region [Fig. 3(b)], making the measurement of $\boldsymbol{\Gamma}$ meaningless here [16]. This reflects the poor correlation between $n_{1}$ and $\Phi_{1}$ in the time-space domain, which is evident in Figs. 1(e) and 1(f) for the time traces measured at $r=12 \mathrm{~cm}$.
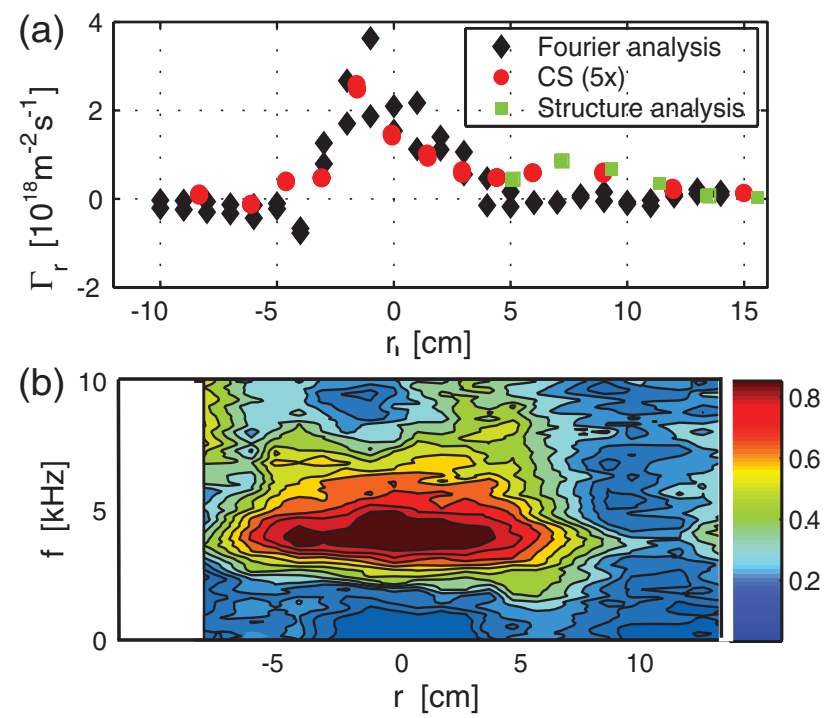

FIG. 3 (color). (a) Radial component of the fluctuationinduced particle flux at midplane calculated through Fourier and CS analysis. The average error, including shot-to-shot variations, is $30 \%$ and $25 \%$, respectively. The average transport associated with propagating structures, identified as blobs for $r \gtrsim 5 \mathrm{~cm}$, is also shown. The average error is $20 \%$. (b) Coherence between $n_{1}$ and $\Phi_{1}$ at the midplane.
Despite the negligible fluctuation-induced flux measured through the Fourier analysis for $r \geq 5 \mathrm{~cm}$, the density decreases in the source-free region, indicating that other mechanisms, viz., the flow along the open magnetic field lines and the blob-related transport, may be responsible for the particle losses. In fact, a nonzero flux already emerges, between $r \approx 5 \mathrm{~cm}$ and $r \approx 10 \mathrm{~cm}$, from the CS analysis [Fig. 3(a)]. When a trigger signal in the main plasma region is used, no transport is measured for $r \geq$ $5 \mathrm{~cm}$, consistently with the Fourier analysis. To investigate the transport associated with blobs, the structure analysis described above is used. The statistical analysis of structure dynamics is based on ion-saturation current data from an 86-tip Langmuir probe array, covering the whole crosssection with a spatial resolution of $3.5 \mathrm{~cm}$. The threshold level defining a structure is $0.1 \times \sigma_{\text {tot }}$, where $\sigma_{\text {tot }}^{2}=$ $(1 / M N) \sum_{i=1}^{M} \sum_{j=1}^{N} \delta n_{i j}^{2} . \delta n_{i j}$ is the $M \times N$ matrix of the density signals, measured in the same discharge over the cross-section. Only structures lasting more than $40 \mu \mathrm{s}$ are retained in the analysis. The low level of the threshold is optimized to enhance the statistics of structures detected at the LFS. As a drawback, the results for the particle transport in the main plasma are dominated by noise, and only results for $r>5 \mathrm{~cm}$ are considered in the following.

The radial particle transport at midplane associated with the macroscopic structures [Eq. (4)] is shown in Fig. 3(a), and the complete 2D pattern is detailed in Fig. 4(a). In the main plasma, and for higher values of $\sigma_{\text {tot }}$, the structure analysis captures the dynamics of macroscopic density perturbations associated with the interchange instability, which appear in the time-space domain as macroscopic cells convected upward by the $\mathbf{E} \times \mathbf{B}$ drift [cf. Figs. 1(c) and 1(d)] [20]. At the far LFS the structures are identified as blobs, leading to bursty, positive-defined density perturbations propagating with a finite radial velocity [Fig. 4(b)]. The associated flux is radially outward, with a finite vertical component for $r>10 \mathrm{~cm}$ [see inset in Fig. 4(a)]. We find $\Gamma_{\text {str }} \lesssim 10^{18} \mathrm{~m}^{-2} \mathrm{~s}^{-1}$, indicating that in the source-free
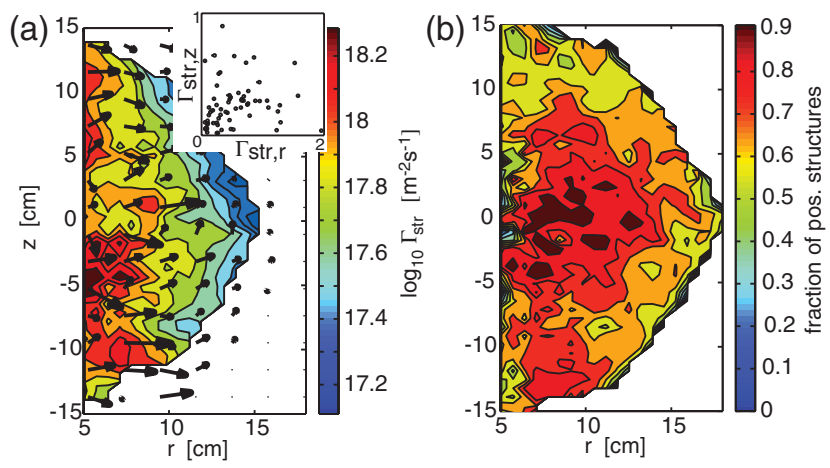

FIG. 4 (color). (a) 2D profile of the instantaneous structurerelated flux, associated with blobs for $r \geq 5 \mathrm{~cm}$. Arrows indicate the direction of propagation of structures. The inset shows the vertical and radial components of the flux (arbitrary units). (b) Fraction of positive structures (normalized to 1). 
region the radial transport is due to the intermittent ejection of blobs. For the experiment described herein, $\sim 10^{2}$ blobs with typical duration $\sim 300 \mu \mathrm{s}$ are observed during $800 \mathrm{~ms}$. The corresponding time-averaged transport is $\$ 4 \%$ of $\Gamma_{\text {str }}$, i.e., it contributes for $\$ 1 \%$ to the total losses. A similar fraction may already translate on fusion-oriented devices into large heat losses, which can eventually damage in-vessel components.

The blob dynamics observed in TORPEX is tightly correlated with the instantaneous $\mathbf{v}_{E \times B}=\mathbf{v}_{E_{0} \times B}+\mathbf{v}_{E_{p} \times B}$ [14], to which two terms contribute. $\mathbf{v}_{E_{0} \times B}$ comes from the time-averaged electric field, and is essentially vertical for the experiments described herein [Fig. 1(b)]. According to theory $[7,8], \mathbf{v}_{E_{p} \times B}$ results from the $\nabla B$-induced polarization of the blob, giving rise to a radial velocity component. We find $\left|v_{E_{0} \times B}\right| \sim\left|v_{E_{p} \times B}\right|$ for $r>10 \mathrm{~cm}$, consistently with the observed blob trajectories. The coupled dynamics of $n$ and $\Phi$, hence $\mathbf{E}$, is the crucial element determining blob dynamics and the associated transport. For the experiments discussed herein, the blob and the associated $\Phi$ perturbation inherit a relative phase shift from the interchange mode, which sets an initial condition for the blob motion. During their propagation, blobs slow down [12] and the coherence between $n$ and $\Phi$ is gradually lost, due to dissipation along $\mathbf{B}$.

Clear differences appear between the transport measured through the different methods. Each technique captures specific aspects of the physics underlying the turbulent flux. The fluctuation-induced flux is by definition a second-order quantity, resulting from linear and local perturbations $n_{1}$ and $\Phi_{1}$, with $\left\langle n_{1}\right\rangle_{t}=\left\langle\Phi_{1}\right\rangle_{t} \equiv 0$. A net transport is found if $n_{1}$ and $\Phi_{1}$ dynamics are coupled, as in the case of fluctuations satisfying a local dispersion relation and with a phase difference $\alpha_{n, \Phi}(\omega) \neq 0, \pm \pi$. Similar considerations hold for the heat flux. These conditions of locality and linearity are only marginally fulfilled by nonlinear, intermittent and macroscopic events such as the blobs, and a different approach is required to characterize the associated transport. For example, the asymmetry between the amount of positive and negative structures, associated with blobs [cf. Fig. 4(b)], is clearly incompatible with a perturbative treatment of fluctuations, typical of the Fourier analysis.

On this basis, instabilities and blobs constitute different mechanisms for anomalous cross-field transport, although the latter is ultimately related to the coupled dynamics of $n$ and $\Phi$ for both phenomena. This results, along with the observed relationship between waves and blobs $[12,14]$, in similar statistical properties in the main plasma and in the source-free region [23]. The specific physics behind fluctuations and blobs naturally translates into different requirements for their experimental study. While singlepoint measurements are sufficient to study fluctuations, understanding structure dynamics requires additional information on the macroscopic density behavior. This can be gathered from probe arrays [12] and conditional sampling techniques [20,21]. In fusion-oriented experiments, the latter could be coupled to fast camera imaging [9], as probes can only span a few centimeters inside the edge plasma. Note that the CS method, which intrinsically selects events correlated with a specific trigger condition, can be considered as an intermediate step between Fourier and structure analysis. An example of combined local and macroscopic analysis has been presented in this Letter. For most experiments a plethora of instabilities, possibly with a turbulent character, may coexist with blobs in the same plasma region. An adequate quantification of the total turbulent flux can be achieved only by combining different methods, providing information on both the linear, local flux and the nonlinear, nonlocal flux associated with blobs.

This work is partly funded by the Swiss National Science Foundation. P.R. is supported by a Euratom Fusion grant.

*mpodesta@pppl.gov

Present address: University of California, Irvine, CA92697, USA.

${ }^{\dagger}$ Present address: University of Warwick, Coventry, CV47AL UK.

${ }^{\star}$ Present address: University of California, San Diego, CA92093, USA.

[1] B. A. Carreras, J. Nucl. Mater. 337, 315 (2005).

[2] G. Y. Antar et al., Phys. Plasmas 10, 419 (2003).

[3] B. Labit et al., Phys. Rev. Lett. 98, 255002 (2007).

[4] E. J. Powers, Nucl. Fusion 14, 749 (1974).

[5] S. J. Zweben, Phys. Fluids 28, 974 (1985).

[6] D. D. Ryutov, Phys. Plasmas 13, 122307 (2006).

[7] O.E. Garcia, V. Naulin, A.H. Nielsen, and J. J. Rasmussen, Phys. Scr. T122, 89 (2006).

[8] S. I. Krasheninnikov, Phys. Lett. A 283, 368 (2001).

[9] S. Zweben et al., Plasma Phys. Controlled Fusion 49, S1 (2007).

[10] J. L. Terry et al., Phys. Plasmas 10, 1739 (2003).

[11] J. R. Myra, D. D'Ippolito, S. I. Krasheninnikov, and G. Q. Yu, Phys. Plasmas 11, 4267 (2004).

[12] S. H. Müller et al., Phys. Plasmas 14, 110704 (2007).

[13] F. M. Poli, P. Ricci, A. Fasoli, and M. Podestà, Phys. Plasmas 15, 032104 (2008).

[14] I. Furno et al., Phys. Rev. Lett. 100, 055004 (2008).

[15] M. Podestá, Ph.D. thesis, Ecole Polythenique Federale de Lausanne, 2007, http://library.epfl.ch/en/theses/?nr=3765.

[16] R. J. Bickerton, Nucl. Fusion 13, 290 (1973).

[17] D. W. Ross, Plasma Phys. Controlled Fusion 34, 137 (1992).

[18] S. H. Müller et al., Phys. Plasmas 13, 100701 (2006).

[19] A. Fasoli et al., Phys. Plasmas 13, 055902 (2006).

[20] I. Furno et al., Phys. Plasmas 15, 055903 (2008).

[21] T. Pierre et al., Phys. Rev. Lett. 92, 065004 (2004).

[22] D. Block, Phys. Scr. T122, 25 (2006).

[23] B. Labit et al., Plasma Phys. Controlled Fusion 49, B281 (2007). 